\title{
SUPPORTING INFORMATION: Using Diffusion
}

\section{Monte Carlo Wave Functions to Analyze the Vibrational Spectra of $\mathrm{H}_{7} \mathrm{O}_{3}{ }^{+}$and $\mathrm{H}_{9} \mathrm{O}_{4}^{+}$}

\author{
Ryan J. DiRisio, ${ }^{\dagger}$ Jacob M. Finney, ${ }^{\dagger}$ Laura C. Dzugan, ${ }^{\dagger}$ Lindsey R. Madison,,${ }^{\ddagger}$ \\ and Anne B. McCoy ${ }^{*, \dagger}$ \\ $\dagger$ Department of Chemistry, University of Washington, Seattle, WA 98195, USA \\ $\ddagger$ Department of Chemistry, Colby College, Waterville, ME 04901, USA \\ E-mail: abmccoy@uw.edu
}

Phone: 206-543-7464 
CONTENTS:

- Numerical Details

- Coordinate Labels for $\mathrm{H}_{7} \mathrm{O}_{3}{ }^{+}$and $\mathrm{H}_{9} \mathrm{O}_{4}^{+}$

- Figure S1: Comparison of Uncoupled, Coupled, and Shifted Coupled GSPA Spectra for All Systems.

- Table S1: Frequencies, Relative Intensities, and Assignments for the GSPA calculation of $\mathrm{H}_{7} \mathrm{O}_{3}{ }^{+}$and $\mathrm{D}_{7} \mathrm{O}_{3}{ }^{+}$.

- Table S2: Frequencies, Relative Intensities, and Assignments for the GSPA calculation of $\mathrm{H}_{9} \mathrm{O}_{4}{ }^{+}$and $\mathrm{D}_{9} \mathrm{O}_{4}{ }^{+}$.

- Table S3: Shifts Applied to the States Involving the Umbrella Mode for All Systems.

- Table S4: Uncoupled GSPA Assignments for the Shared Proton Stretch Region of $\mathrm{H}_{9} \mathrm{O}_{4}{ }^{+}$.

- Table S5: Uncoupled GSPA Assignments for the Shared Proton Stretch Region of $\mathrm{D}_{9} \mathrm{O}_{4}^{+}$.

- Table S6: Uncoupled GSPA Assignments for the Shared Proton Stretch Region of $\mathrm{H}_{7} \mathrm{O}_{3}{ }^{+}$.

- Table S7: Uncoupled GSPA Assignments for the Shared Proton Stretch Region of $\mathrm{D}_{7} \mathrm{O}_{3}{ }^{+}$.

- Table S8: Shifted, Coupled GSPA Assignments for the Shared Proton Stretch Region of $\mathrm{H}_{9} \mathrm{O}_{4}^{+}$.

- Table S9: Shifted, Coupled GSPA Assignments for the Shared Proton Stretch Region of $\mathrm{D}_{9} \mathrm{O}_{4}{ }^{+}$. 
- Table S10: Shifted, Coupled GSPA Assignments for the Shared Proton Stretch Region of $\mathrm{H}_{7} \mathrm{O}_{3}{ }^{+}$.

- Table S11: Shifted, Coupled GSPA Assignments for the Shared Proton Stretch Region of $\mathrm{D}_{7} \mathrm{O}_{3}{ }^{+}$.

- Table S12: Coupled GSPA Assignments for the Shared Proton Stretch Region of $\mathrm{H}_{9} \mathrm{O}_{4}{ }^{+}$.

- Table S13: Coupled GSPA Assignments for the Shared Proton Stretch Region of $\mathrm{D}_{9} \mathrm{O}_{4}^{+}$.

- Table S14: Coupled GSPA Assignments for the Shared Proton Stretch Region of $\mathrm{H}_{7} \mathrm{O}_{3}{ }^{+}$.

- Table S15: Coupled GSPA Assignments for the Shared Proton Stretch Region of $\mathrm{D}_{7} \mathrm{O}_{3}{ }^{+}$.

- Table S16: Eigenvectors of Degenerate VPT2 Mixed States For Intense Features in the Shared Proton Stretch Region of $\mathrm{H}_{7} \mathrm{O}_{3}{ }^{+}$.

- Table S17: Eigenvectors of Degenerate VPT2 Mixed States For Intense Features in the Shared Proton Stretch Region of $\mathrm{D}_{7} \mathrm{O}_{3}{ }^{+}$.

- Table S18: Eigenvectors of Degenerate VPT2 Mixed States For Intense Features in the Shared Proton Stretch Region of $\mathrm{H}_{9} \mathrm{O}_{4}{ }^{+}$.

- Table S19: Uncoupled GSPA Assignments for the Low Frequency Region of $\mathrm{H}_{7} \mathrm{O}_{3}{ }^{+}$ and $\mathrm{H}_{9} \mathrm{O}_{4}{ }^{+}$. 


\section{Numerical Details}

The parameters of the DMC simulations of $\mathrm{H}_{7} \mathrm{O}_{3}{ }^{+}, \mathrm{D}_{7} \mathrm{O}_{3}{ }^{+}, \mathrm{H}_{9} \mathrm{O}_{4}{ }^{+}$, and $\mathrm{D}_{9} \mathrm{O}_{4}{ }^{+}$are reported in a previous study. ${ }^{1}$ The DMC wave functions and descendant weights were collected from five independent 30000 walker simulations for $\mathrm{H}_{7} \mathrm{O}_{3}{ }^{+}$and $\mathrm{D}_{7} \mathrm{O}_{3}{ }^{+}$and 20000 walker simulations for $\mathrm{H}_{9} \mathrm{O}_{4}{ }^{+}$and $\mathrm{D}_{9} \mathrm{O}_{4}{ }^{+}$. In that work, several forms of $\Psi_{\mathrm{T}}$ were used, and we employ the wave functions that were obtained using the $\Psi_{\mathrm{T}}$ function that was developed for the system that is being described.

The wave functions that are obtained from DMC often do not fully reflect the symmetry of the system being studied. To correct for this, the DMC wave functions were symmetrized to ensure that the appropriate symmetry is reflected by the ground state probability amplitude. This is achieved by performing the appropriate symmetry operations on each walker, and in this way generating 15 new walkers for each walker that describes $\mathrm{H}_{7} \mathrm{O}_{3}{ }^{+}$and $\mathrm{D}_{7} \mathrm{O}_{3}{ }^{+}$and 47 new walkers for each walker in the ensembles describing $\mathrm{H}_{9} \mathrm{O}_{4}{ }^{+}$and $\mathrm{D}_{9} \mathrm{O}_{4}{ }^{+}$. There are three symmetries that are considered. First, the wave function must be symmetric with respect to the two hydrogen atoms on any of the water molecules. It must also be symmetric with respect to exchange of any $\mathrm{H} \cdot \mathrm{OH}_{2}$ group where the first $\mathrm{H}$ is one of the hydrogen-bonded hydrogen atoms in the hydronium core, and $\mathrm{OH}_{2}$ represents thee bound water molecule. Finally, the wave function must be symmetric with respect to reflection in the $A B$ plane (see Figure 2).

\section{Coordinate Labels for $\mathrm{H}_{7} \mathrm{O}_{3}^{+}$and $\mathrm{H}_{9} \mathrm{O}_{4}^{+}$}

It is useful to establish the notation that will be used to describe the transitions of interest in $\mathrm{H}_{7} \mathrm{O}_{3}{ }^{+}$and $\mathrm{H}_{9} \mathrm{O}_{4}{ }^{+}$. In the following tables, b, f, and w superscripts indicate if the hydrogen atom involved in the vibrational motion is associated with a flanking water molecule (denoted as $\mathrm{w}$ ), involved in a hydrogen bond between the hydronium core and a water molecule (denoted as b) or, in the case of $\mathrm{H}_{7} \mathrm{O}_{3}{ }^{+}$, a hydrogen atom in the hydronium core that is not 
involved in making a hydrogen bond (denoted as f). Additionally, the superscripts s and a are used to denote symmetric and antisymmetric combinations of $\mathrm{OH}$ vibrations in the same molecular unit, while $\mathrm{i}$ and o are used to denote in- or out-of-phase combinations of vibrations on two or more of the flanking water molecules. For intramolecular vibrations and stretching motions, subscripts are used to indicate which atoms are involved in the described motions, e.g. $\mathrm{OH}$ for an $\mathrm{OH}$-stretching vibration or $\mathrm{HOH}$ for an $\mathrm{HOH}-$ bending vibration. For intermolecular vibrations the subscripts are used to describe the type of motion, e.g. wag, rock or rot(ation). In the case of rotation of the $\mathrm{H}_{3} \mathrm{O}^{+}$core, the rotation axis is also specified, and the orientation of the axis is based on the modified Eckart embedding described above. While these definitions provide the leading contributions to the coordinates that are obtained by diagonalizing the mass-weighted matrix of second moments, they provide good zero-order descriptions of the associated vibrations. The energies and relative intensities associated with the transitions to states with one quantum of excitation in each of these modes obtained using the GSPA approach are provided in Tables S1 and S2 for $\mathrm{H}_{7} \mathrm{O}_{3}{ }^{+}$and $\mathrm{H}_{9} \mathrm{O}_{4}{ }^{+}$and their deuterated analogues, respectively. 


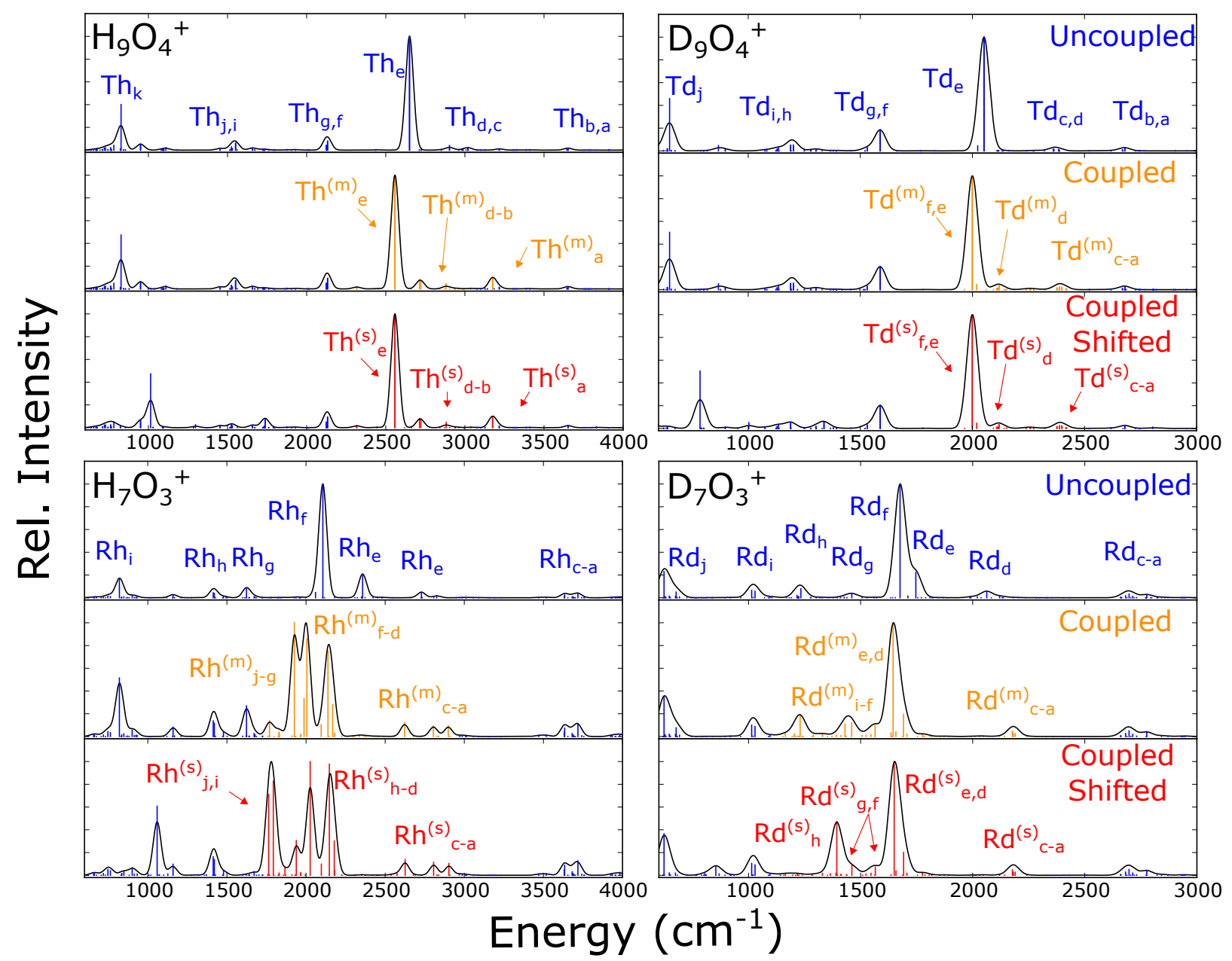

Figure S1: Calculated uncoupled GSPA (blue sticks, top), coupled GSPA (gold sticks, middle), and shifted coupled GSPA (red sticks, bottom) spectra of $\mathrm{H}_{7} \mathrm{O}_{3}{ }^{+}$(left) $\mathrm{D}_{7} \mathrm{O}_{3}{ }^{+}$(right). In these spectra, the Th and Rh labels are used to denote transitions in $\mathrm{H}_{9} \mathrm{O}_{4}{ }^{+}$and $\mathrm{H}_{7} \mathrm{O}_{3}{ }^{+}$, respectively, while $\mathrm{Td}$ and $\mathrm{Rd}$ are used to refer to peaks in the spectra of the deuterated forms of these ions. For the shifted GSPA spectra, the energies of the transitions involving the umbrella mode have been shifted by the amounts provided in Table S3. Assignments for the labeled transitions in the uncoupled spectra are provided in Tables S4-S7, assignments of the transitions in the coupled spectra are provided in Tables S12-S15, and the assignments of the transitions in the coupled shifted spectra can be found in S8-S11. 


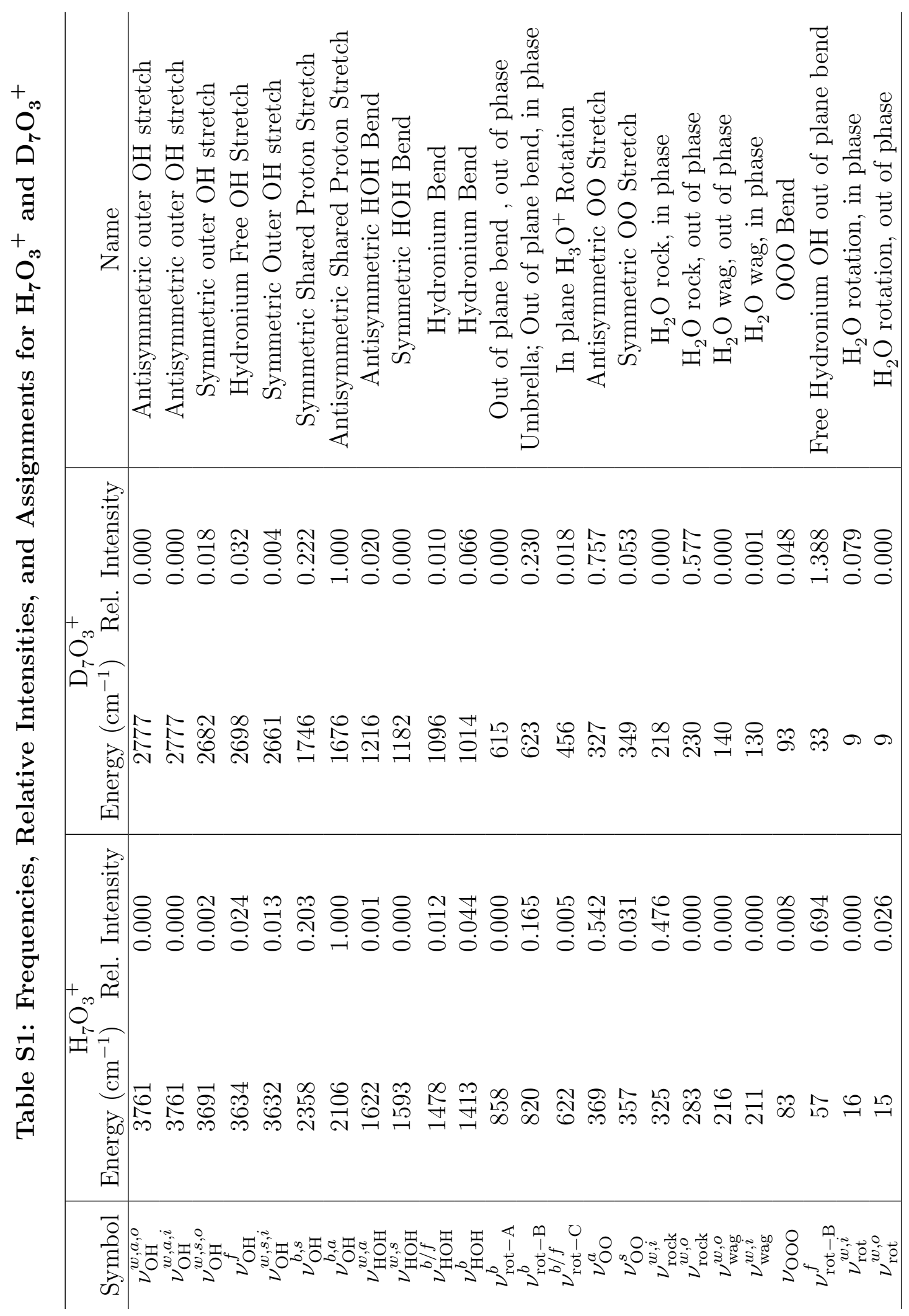




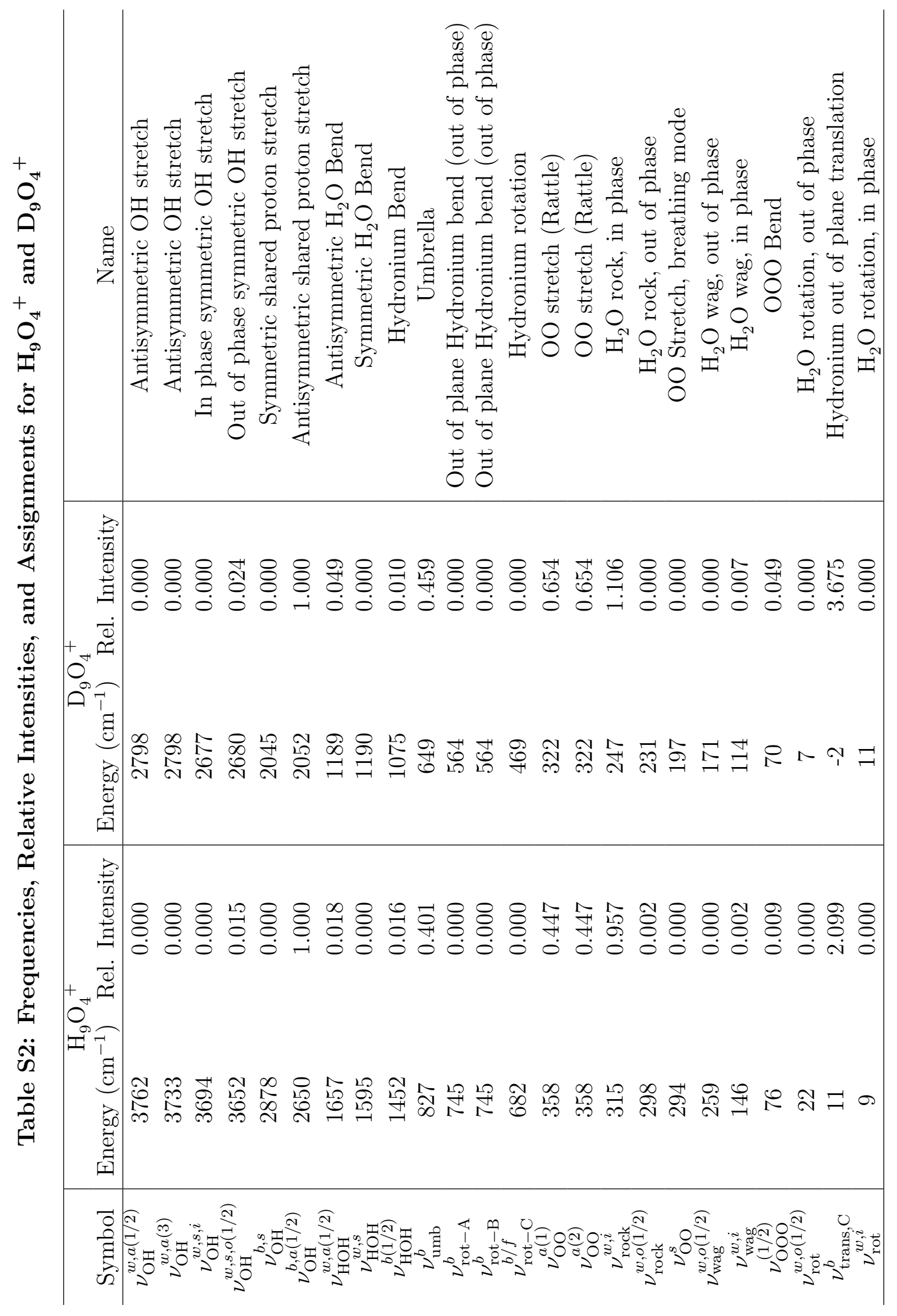


Table S3: Shifts Applied to the Transitions Involving the Umbrella mode for $\mathrm{H}_{7} \mathrm{O}_{3}{ }^{+}, \mathrm{D}_{7} \mathrm{O}_{3}{ }^{+}, \mathrm{H}_{9} \mathrm{O}_{4}{ }^{+}$, and $\mathrm{D}_{9} \mathrm{O}_{4}{ }^{+}$Based on the $\Delta n=1$ Transition Energies $\left(\mathrm{cm}^{-1}\right)$

\begin{tabular}{lccc}
\hline System & GSPA & Measured & Shift \\
\hline $\mathrm{H}_{7} \mathrm{O}_{3}{ }^{+}$ & 820 & $1059^{a}$ & 239 \\
$\mathrm{D}_{7} \mathrm{O}_{3}{ }^{+}$ & 623 & $800^{a}$ & 177 \\
$\mathrm{H}_{9} \mathrm{O}_{4}^{+}$ & 827 & $1014^{b}$ & 187 \\
$\mathrm{D}_{9} \mathrm{O}_{4}^{+}$ & 649 & - & $138^{c}$ \\
\hline${ }^{a}$ Ref. 2. \\
${ }^{b}$ Ref. 3. \\
${ }^{c}$ Calculated based on the other shifts \\
using $177 / 239 \times 187=138$.
\end{tabular}


Table S4: Uncoupled GSPA Calculated Frequencies, Relative Intensities, and Assignments for the Shared Proton Stretch Region of $\mathrm{H}_{9} \mathrm{O}_{4}^{+a}$ (Th)

\begin{tabular}{|c|c|c|c|}
\hline Symbol & Frequency $\left(\mathrm{cm}^{-1}\right)$ & Rel. Int. & Assignment \\
\hline \multirow[t]{2}{*}{$\mathrm{Th}_{\mathrm{a}}$} & 3652 & 0.015 & $\nu_{\mathrm{OH}}^{w, s, o(2)}$ \\
\hline & 3652 & 0.015 & $\nu_{\mathrm{OH}}^{w, s, o(1)}$ \\
\hline $\mathrm{Th}_{\mathrm{b}}$ & 3644 & 0.005 & $\nu_{\mathrm{OH}}^{b, s}+\nu_{\mathrm{umb}}^{b}$ \\
\hline \multirow[t]{6}{*}{$\mathrm{Th}_{\mathrm{c}}$} & 3019 & 0.011 & $\nu_{\mathrm{OH}}^{b, a(2)}+\nu_{\mathrm{OO}}^{a(1)}$ \\
\hline & 3019 & 0.011 & $\nu_{\mathrm{OH}}^{b, a(1)}+\nu_{\mathrm{OO}}^{a(2)}$ \\
\hline & 3018 & 0.011 & $\nu_{\mathrm{OH}}^{b, a(1)}+\nu_{\mathrm{OO}}^{a(1)}$ \\
\hline & 3018 & 0.011 & $\nu_{\mathrm{OH}}^{b, a(2)}+\nu_{\mathrm{OO}}^{a(2)}$ \\
\hline & 3217 & 0.011 & $\nu_{O H}^{b, s}+\nu_{O O}^{a(2)}$ \\
\hline & 3217 & 0.011 & $\nu_{\mathrm{OH}}^{b, s}+\nu_{\mathrm{OO}}^{a(1)}$ \\
\hline $\mathrm{Th}_{\mathrm{d}}$ & 2902 & 0.041 & $\nu_{\mathrm{OH}}^{b, s}+\nu_{\text {trans }, \mathrm{C}}^{b}$ \\
\hline \multirow[t]{2}{*}{$\mathrm{Th}_{\mathrm{e}}$} & 2650 & 1.000 & $\nu_{\mathrm{OH}}^{b, a(2)}$ \\
\hline & 2650 & 1.000 & $\nu_{\mathrm{OH}}^{b, a(1)}$ \\
\hline \multirow[t]{2}{*}{$\mathrm{Th}_{\mathrm{f}}$} & 2132 & 0.078 & $\nu_{\mathrm{HOH}}^{b(2)}+\nu_{\mathrm{rot}-\mathrm{B}}^{b}$ \\
\hline & 2132 & 0.078 & $\nu_{\mathrm{HOH}}^{b(1)}+\nu_{\mathrm{rot}-\mathrm{A}}^{b}$ \\
\hline \multirow[t]{2}{*}{$\mathrm{Th}_{\mathrm{g}}$} & 2124 & 0.041 & $\nu_{\mathrm{HOH}}^{b(2)}+\nu_{\mathrm{rot}-\mathrm{C}}^{b / f}$ \\
\hline & 2124 & 0.041 & $\nu_{\mathrm{HOH}}^{b(1)}+\nu_{\mathrm{rot}-\mathrm{C}}^{b / f}$ \\
\hline \multirow[t]{2}{*}{$\mathrm{Th}_{\mathrm{h}}$} & 1657 & 0.018 & $\nu_{\mathrm{HOH}}^{w, a(1)}$ \\
\hline & 1657 & 0.018 & $\nu_{\mathrm{HOH}}^{w, a(2)}$ \\
\hline \multirow[t]{2}{*}{$\mathrm{Th}_{\mathrm{i}}$} & 1551 & 0.061 & $\nu_{\mathrm{umb}}^{b}+\nu_{\mathrm{rot}-\mathrm{A}}^{b}$ \\
\hline & 1551 & 0.061 & $\nu_{\mathrm{umb}}^{b}+\nu_{\mathrm{rot}-\mathrm{B}}^{b}$ \\
\hline \multirow[t]{3}{*}{$\mathrm{Th}_{\mathrm{j}}$} & 1525 & 0.028 & $\nu_{\mathrm{rot}-\mathrm{A}}^{b}+\nu_{\mathrm{rot}-\mathrm{B}}^{b}$ \\
\hline & 1452 & 0.016 & $\nu_{\mathrm{HOH}}^{b(1)}$ \\
\hline & 1452 & 0.016 & $\nu_{\mathrm{HOH}}^{b(2)}$ \\
\hline $\mathrm{Th}_{\mathrm{k}}$ & 827 & 0.401 & $\nu_{\mathrm{umb}}^{b}$ \\
\hline
\end{tabular}


Table S5: Uncoupled GSPA Calculated Frequencies, Relative Intensities, and Assignments for the Shared Proton Stretch Region of $\mathrm{D}_{9} \mathrm{O}_{4}{ }^{+a}$ (Td)

\begin{tabular}{|c|c|c|c|}
\hline Symbol & Frequency $\left(\mathrm{cm}^{-1}\right)$ & Rel. Int. & Assignment \\
\hline \multirow[t]{2}{*}{$\mathrm{Td}_{\mathrm{a}}$} & 2680 & 0.024 & $\nu_{\mathrm{OH}}^{w, s, o(1)}$ \\
\hline & 2680 & 0.024 & $\nu_{\mathrm{OH}}^{w, s, o(2)}$ \\
\hline $\mathrm{Td}_{\mathrm{b}}$ & 2669 & 0.011 & $\nu_{\mathrm{OH}}^{b, s}+\nu_{\mathrm{umb}}^{b}$ \\
\hline \multirow[t]{2}{*}{$\mathrm{Td}_{\mathrm{c}}$} & 2385 & 0.011 & $\nu_{\mathrm{OH}}^{b, a(2)}+\nu_{\mathrm{OO}}^{a(2)}$ \\
\hline & 2385 & 0.011 & $\nu_{\mathrm{OH}}^{b, a(1)}+\nu_{\mathrm{OO}}^{a(1)}$ \\
\hline \multirow[t]{4}{*}{$\mathrm{Td}_{\mathrm{d}}$} & 2363 & 0.013 & $\nu_{\mathrm{OH}}^{b, s}+\nu_{\mathrm{OO}}^{a(1)}$ \\
\hline & 2363 & 0.013 & $\nu_{\mathrm{OH}}^{b, s}+\nu_{\mathrm{O}}^{a(2)}$ \\
\hline & 2361 & 0.011 & $\nu_{\mathrm{OH}}^{b, a(1)}+\nu_{\mathrm{OO}}^{a(2)}$ \\
\hline & 2361 & 0.011 & $\nu_{\mathrm{OH}}^{b, a(2)}+\nu_{\mathrm{OO}}^{a(1)}$ \\
\hline \multirow[t]{2}{*}{$\mathrm{Td}_{\mathrm{e}}$} & 2052 & 1.000 & $\nu_{\mathrm{OH}}^{b, a(2)}$ \\
\hline & 2052 & 1.000 & $\nu_{\mathrm{OH}}^{b, a(1)}$ \\
\hline \multirow[t]{2}{*}{$\mathrm{Td}_{\mathrm{f}}$} & 1589 & 0.185 & $\nu_{\mathrm{HOH}}^{b(1)}+\nu_{\mathrm{rot}-\mathrm{A}}^{b}$ \\
\hline & 1589 & 0.185 & $\nu_{\mathrm{HOH}}^{b(2)}+\nu_{\mathrm{rot}-\mathrm{B}}^{b}$ \\
\hline \multirow[t]{2}{*}{$\mathrm{Td}_{\mathrm{g}}$} & 1530 & 0.027 & $\nu_{\mathrm{HOH}}^{b(2)}+\nu_{\mathrm{rot}-\mathrm{C}}^{b / f}$ \\
\hline & 1530 & 0.027 & $\nu_{\mathrm{HOH}}^{b(1)}+\nu_{\mathrm{rot}-\mathrm{C}}^{b / f}$ \\
\hline \multirow[t]{2}{*}{$\mathrm{Td}_{\mathrm{h}}$} & 1202 & 0.050 & $\nu_{\mathrm{umb}}^{b}+\nu_{\mathrm{rot}-\mathrm{A}}^{b}$ \\
\hline & 1202 & 0.050 & $\nu_{\mathrm{umb}}^{b}+\nu_{\mathrm{rot}-\mathrm{B}}^{b}$ \\
\hline \multirow[t]{2}{*}{$\mathrm{Td}_{\mathrm{i}}$} & 1189 & 0.049 & $\nu_{\mathrm{HOH}}^{w, a(2)}$ \\
\hline & 1189 & 0.049 & $\nu_{\mathrm{HOH}}^{w, a(1)}$ \\
\hline $\mathrm{Td}_{\mathrm{j}}$ & 649 & 0.459 & $\nu_{u m b}^{b}$ \\
\hline
\end{tabular}


Table S6: Uncoupled GSPA Calculated Frequencies, Relative Intensities, and Assignments for the Shared Proton Stretch Region of $\mathrm{H}_{7} \mathrm{O}_{3}{ }^{+a}(\mathrm{Rh})$

\begin{tabular}{cccc}
\hline Symbol & Frequency $\left(\mathrm{cm}^{-1}\right)$ & Rel. Int. & Assignment \\
\hline $\mathrm{Rh}_{\mathrm{a}}$ & 3718 & 0.034 & $\nu_{\mathrm{OH}}^{b, s}+\nu_{\mathrm{HOH}}^{b / f}$ \\
$\mathrm{Rh}_{\mathrm{b}}$ & 3682 & 0.008 & $\nu_{\mathrm{OH}}^{f}+\nu_{\mathrm{rot}-\mathrm{B}}^{f}$ \\
& 3688 & 0.004 & $\nu_{\mathrm{OH}}^{b, a}+\nu_{\mathrm{HOH}}^{w, s}$ \\
$\mathrm{Rh}_{\mathrm{c}}$ & 3634 & 0.024 & $\nu_{\mathrm{OH}}^{f}$ \\
& 3632 & 0.013 & $\nu_{\mathrm{OH}}^{w, s, i}$ \\
$\mathrm{Rh}_{\mathrm{d}}$ & 2729 & 0.050 & $\nu_{\mathrm{OH}}^{b, s}+\nu_{\mathrm{OO}}^{a}$ \\
& 2826 & 0.016 & $\nu_{\mathrm{HOH}}^{b f}+\nu_{\mathrm{HOH}}^{b}$ \\
$\mathrm{Rh}_{\mathrm{e}}$ & 2358 & 0.203 & $\nu_{\mathrm{OH}}^{b, s}$ \\
$\mathrm{Rh}_{\mathrm{f}}$ & 2106 & 1.000 & $\nu_{\mathrm{OH}}^{b, a}$ \\
& 2060 & 0.046 & $\nu_{\mathrm{HOH}}^{b}+\nu_{\mathrm{rot}-\mathrm{C}}^{b / f}$ \\
$\mathrm{Rh}_{\mathrm{g}}$ & 1624 & 0.086 & $\nu_{\mathrm{rot}-\mathrm{A}}^{b}+\nu_{\mathrm{rot}-\mathrm{B}}^{b}$ \\
$\mathrm{Rh}_{\mathrm{h}}$ & 1413 & 0.044 & $\nu_{\mathrm{HOH}}^{b}$ \\
& 1421 & 0.037 & $\nu_{\mathrm{HOH}}^{b}+\nu_{\mathrm{rot}-\mathrm{B}}^{f}$ \\
& 1478 & 0.012 & $\nu_{\mathrm{HOH}}^{b / f}$ \\
$\mathrm{Rh}_{\mathrm{i}}$ & 820 & 0.165 & $\nu_{\mathrm{rot}-\mathrm{B}}^{b}$ \\
\hline$a \mathrm{These}$ features are plotted in blue in Figures 3 and S1.
\end{tabular}


Table S7: Uncoupled GSPA Calculated Frequencies, Relative Intensities, and Assignments for the Shared Proton Stretch Region of $\mathrm{D}_{7} \mathrm{O}_{3}{ }^{+a}$ ( $\left.\mathrm{Rd}\right)$

\begin{tabular}{|c|c|c|c|}
\hline Symbol & Frequency $\left(\mathrm{cm}^{-1}\right)$ & Rel. Int. & Assignment \\
\hline $\operatorname{Rd}_{\mathrm{a}}$ & 2777 & 0.028 & $\nu_{\mathrm{OH}}^{b, s}+\nu_{\mathrm{HOH}}^{b / f}$ \\
\hline $\mathrm{Rd}_{\mathrm{b}}$ & 2714 & 0.012 & $\nu_{\mathrm{OH}}^{f}+\nu_{\mathrm{rot}-\mathrm{B}}^{f}$ \\
\hline \multirow[t]{2}{*}{$\mathrm{Rd}_{\mathrm{c}}$} & 2698 & 0.032 & $\nu_{\mathrm{OH}}^{f}$ \\
\hline & 2682 & 0.018 & $\nu_{\mathrm{OH}}^{w, s, o}$ \\
\hline $\operatorname{Rd}_{\mathrm{d}}$ & 2063 & 0.056 & $\nu_{\mathrm{OH}}^{b, s}+\nu_{\mathrm{OO}}^{a}$ \\
\hline $\operatorname{Rd}_{\mathrm{r}}$ & 1746 & 0.222 & $\nu_{\mathrm{OH}}^{b, s}$ \\
\hline $\operatorname{Rd}_{\mathrm{f}}$ & 1676 & 1.000 & $\nu_{\mathrm{OH}}^{b, a}$ \\
\hline $\operatorname{Rd}_{\mathrm{g}}$ & 1461 & 0.036 & $\nu_{\mathrm{HOH}}^{b}+\nu_{\mathrm{rot}-\mathrm{C}}^{b / f}$ \\
\hline $\mathrm{Rd}_{\mathrm{h}}$ & 1234 & 0.079 & $\nu_{\mathrm{rot}-\mathrm{B}}^{b}+\nu_{\mathrm{rot}-\mathrm{A}}^{b}$ \\
\hline \multirow[t]{2}{*}{$\operatorname{Rd}_{\mathrm{i}}$} & 1014 & 0.066 & $\nu_{\mathrm{HOH}}^{b}$ \\
\hline & 1029 & 0.057 & $\nu_{\mathrm{HOH}}^{b}+\nu_{\mathrm{rot}-\mathrm{B}}^{f}$ \\
\hline $\operatorname{Rd}_{j}$ & 623 & 0.230 & $\nu_{\text {rot-B }}^{b}$ \\
\hline
\end{tabular}

${ }^{a}$ These features are plotted in blue in Figures 3 and S1. 
Table S8: Shifted, Coupled GSPA Frequencies, Relative Intensities, and Assignments for $\mathrm{H}_{9} \mathrm{O}_{4}{ }^{+a}$ (Th)

\begin{tabular}{|c|c|c|c|c|}
\hline Symbol & Frequency $\left(\mathrm{cm}^{-1}\right)$ & Rel. Int. & $C_{n}$ & $n$ \\
\hline \multirow[t]{3}{*}{$\mathrm{Th}_{\mathrm{a}}^{(\mathrm{s})}$} & 3175 & 0.098 & -0.530 & $\nu_{\mathrm{OH}}^{b, a(2)}+\nu_{\mathrm{OO}}^{a(2)}$ \\
\hline & & & -0.530 & $\nu_{\mathrm{OH}}^{b, a(1)}+\nu_{\mathrm{OO}}^{a(1)}$ \\
\hline & & & 0.485 & $\nu_{\mathrm{OH}}^{b, a(2)}+\nu_{\mathrm{OO}}^{s}$ \\
\hline \multirow[t]{3}{*}{$\mathrm{Th}_{\mathrm{a}}^{(\mathrm{s})}$} & 3175 & 0.098 & -0.530 & $\nu_{\mathrm{OH}}^{b, a(2)}+\nu_{\mathrm{OO}}^{a(1)}$ \\
\hline & & & 0.530 & $\nu_{\mathrm{OH}}^{b, a(1)}+\nu_{\mathrm{OO}}^{a(2)}$ \\
\hline & & & 0.486 & $\nu_{\mathrm{OH}}^{b, a(1)}+\nu_{\mathrm{OO}}^{s}$ \\
\hline \multirow[t]{3}{*}{$\mathrm{Th}_{\mathrm{b}}^{(\mathrm{s})}$} & 2882 & 0.046 & 0.903 & $\nu_{\mathrm{OH}}^{b, s}+\nu_{\text {trans }, \mathrm{C}}^{b}$ \\
\hline & & & 0.394 & $\nu_{\mathrm{OH}}^{b, s}+\nu_{\mathrm{wag}}^{w, i}$ \\
\hline & & & -0.098 & $\nu_{\mathrm{OH}}^{b, a(2)}+\nu_{\text {rock }}^{w, o(2)}$ \\
\hline \multirow[t]{3}{*}{$\mathrm{Th}_{\mathrm{c}}^{(\mathrm{s})}$} & 2722 & 0.075 & -0.685 & $\nu_{\mathrm{OH}}^{b, a(1)}+\nu_{\mathrm{OO}}^{s}$ \\
\hline & & & -0.675 & $\nu_{\mathrm{OH}}^{b, a(2)}+\nu_{\mathrm{OOO}}^{(2)}$ \\
\hline & & & 0.229 & $\nu_{\mathrm{OH}}^{b, a(1)}$ \\
\hline \multirow[t]{3}{*}{$\mathrm{Th}_{\mathrm{d}}^{(\mathrm{s})}$} & 2713 & 0.081 & 0.676 & $\nu_{\mathrm{OH}}^{b, a(1)}+\nu_{\mathrm{OOO}}^{(2)}$ \\
\hline & & & -0.676 & $\nu_{\mathrm{OH}}^{b, a(2)}+\nu_{\mathrm{OOO}}^{(1)}$ \\
\hline & & & -0.238 & $\nu_{\mathrm{OH}}^{b, a(2)}$ \\
\hline \multirow[t]{3}{*}{$\operatorname{Th}_{\mathrm{e}}^{(\mathrm{s})}$} & 2558 & 1.000 & 0.686 & $\nu_{\mathrm{OH}}^{b, a(1)}$ \\
\hline & & & -0.531 & $\nu_{\mathrm{OH}}^{b, a(2)}$ \\
\hline & & & 0.211 & $\nu_{\mathrm{OH}}^{b, a(2)}+\nu_{\mathrm{OO}}^{a(2)}$ \\
\hline \multirow[t]{3}{*}{$\operatorname{Th}_{\mathrm{e}}^{(\mathrm{s})}$} & 2558 & 0.997 & -0.684 & $\nu_{\mathrm{OH}}^{b, a(2)}$ \\
\hline & & & -0.531 & $\nu_{\mathrm{OH}}^{b, a(1)}$ \\
\hline & & & 0.211 & $\nu_{\mathrm{OH}}^{b, a(2)}+\nu_{\mathrm{OO}}^{a(1)}$ \\
\hline
\end{tabular}

${ }^{a}$ These features are plotted in red in Figure 3 and Figure S1. 
Table S9: Shifted, Coupled GSPA Frequencies, Relative Intensities, and Assignments for $\mathrm{D}_{9} \mathrm{O}_{4}{ }^{+a}(\mathrm{Td})$

\begin{tabular}{|c|c|c|c|c|}
\hline Symbol & Frequency $\left(\mathrm{cm}^{-1}\right)$ & Rel. Int. & $C_{n}$ & $n$ \\
\hline \multirow[t]{3}{*}{$\mathrm{Td}_{\mathrm{a}}^{(\mathrm{s})}$} & 2399 & 0.022 & -0.651 & $\nu_{\mathrm{OH}}^{b, s}+\nu_{\mathrm{OO}}^{a(2)}$ \\
\hline & & & -0.429 & $\nu_{\mathrm{HOH}}^{w, a(1)}+\nu_{\mathrm{HOH}}^{w, s}$ \\
\hline & & & 0.367 & $\nu_{\mathrm{HOH}}^{w, a(2)}+\nu_{\mathrm{HOH}}^{w, a(2)}$ \\
\hline \multirow[t]{3}{*}{$\operatorname{Td}_{\mathrm{b}}^{(\mathrm{s})}$} & 2388 & 0.016 & 0.683 & $\nu_{\mathrm{HOH}}^{w, a(1)}+\nu_{\mathrm{HOH}}^{w, s}$ \\
\hline & & & -0.635 & $\nu_{\mathrm{OH}}^{b, s}+\nu_{\mathrm{OO}}^{a(2)}$ \\
\hline & & & -0.244 & $\nu_{\mathrm{HOH}}^{w, a(2)}+\nu_{\mathrm{HOH}}^{w, a(2)}$ \\
\hline \multirow[t]{3}{*}{$\operatorname{Td}_{\mathrm{c}}^{(\mathrm{s})}$} & 2389 & 0.024 & -0.706 & $\nu_{\mathrm{OH}}^{b, s}+\nu_{\mathrm{OO}}^{a(1)}$ \\
\hline & & & -0.584 & $\nu_{\mathrm{HOH}}^{w, a(2)}+\nu_{\mathrm{HOH}}^{w, s}$ \\
\hline & & & -0.318 & $\nu_{\mathrm{HOH}}^{w, a(1)}+\nu_{\mathrm{HOH}}^{w, a(2)}$ \\
\hline \multirow[t]{3}{*}{$\mathrm{Td}_{\mathrm{d}}^{(\mathrm{s})}$} & 2119 & 0.034 & -0.558 & $\nu_{\mathrm{OH}}^{b, s}+\nu_{\mathrm{OOO}}^{(1)}$ \\
\hline & & & 0.499 & $\nu_{\mathrm{OH}}^{b, a(1)}+\nu_{\mathrm{OOO}}^{(1)}$ \\
\hline & & & -0.498 & $\nu_{\mathrm{OH}}^{b,(2)}+\nu_{\mathrm{OOO}}^{(2)}$ \\
\hline \multirow[t]{3}{*}{$\mathrm{Td}_{\mathrm{d}}^{(\mathrm{s})}$} & 2118 & 0.031 & -0.634 & $\nu_{\mathrm{OH}}^{b, s}+\nu_{\mathrm{OOO}}^{(2)}$ \\
\hline & & & -0.441 & $\nu_{\mathrm{OH}}^{b, a(2)}+\nu_{\mathrm{OOO}}^{(1)}$ \\
\hline & & & 0.394 & $\nu_{\mathrm{HOH}}^{b(2)}+\nu_{\mathrm{HOH}}^{b(2)}$ \\
\hline \multirow[t]{3}{*}{$\mathrm{Td}_{\mathrm{e}}^{(\mathrm{s})}$} & 2019 & 0.045 & 0.977 & $\nu_{\mathrm{OH}}^{b, s}+\nu_{\text {trans, } \mathrm{C}}^{b}$ \\
\hline & & & -0.178 & $\nu_{\mathrm{OH}}^{b, s}+\nu_{\text {wag }}^{w, i}$ \\
\hline & & & 0.094 & $\nu_{\mathrm{HOH}}^{w, s}+\nu_{\mathrm{umb}}^{b}$ \\
\hline \multirow[t]{3}{*}{$\mathrm{Td}_{\mathrm{f}}^{(\mathrm{s})}$} & 2000 & 1.000 & -0.884 & $\nu_{\mathrm{OH}}^{b, a(2)}$ \\
\hline & & & -0.210 & $\nu_{\mathrm{OH}}^{b, s}+\nu_{\mathrm{OO}}^{a(1)}$ \\
\hline & & & -0.167 & $\nu_{\mathrm{HOH}}^{b(1)}+\nu_{\mathrm{HOH}}^{b(2)}$ \\
\hline \multirow[t]{3}{*}{$\mathrm{Td}_{\mathrm{f}}^{(\mathrm{s})}$} & 1999 & 0.994 & 0.881 & $\nu_{\mathrm{OH}}^{b, a(1)}$ \\
\hline & & & -0.210 & $\nu_{\mathrm{OH}}^{b, s}+\nu_{\mathrm{OO}}^{a(2)}$ \\
\hline & & & -0.156 & $\nu_{\mathrm{OH}}^{b, a(2)}+\nu_{\mathrm{OO}}^{a(1)}$ \\
\hline
\end{tabular}

${ }^{a}$ These features are plotted in red in Figure 3 and Figure S1. 
Table S10: Shifted, Coupled GSPA Frequencies, Relative Intensities, and Assignments for $\mathrm{H}_{7} \mathrm{O}_{3}{ }^{+a}$ (Rh)

\begin{tabular}{|c|c|c|c|c|}
\hline Symbol & Frequency $\left(\mathrm{cm}^{-1}\right)$ & Rel. Int. & $C_{n}$ & $n$ \\
\hline $\mathrm{Rh}_{\mathrm{a}}^{(\mathrm{s})}$ & 2903 & 0.104 & $\begin{array}{c}0.908 \\
-0.425 \\
0.294\end{array}$ & $\begin{array}{c}\nu_{\mathrm{OH}}^{b, s}+\nu_{\mathrm{OO}}^{A} \\
\nu_{\mathrm{OH}}^{b, a} \\
\nu_{\mathrm{OH}}^{b, a}+\nu_{\mathrm{OO}}^{s}\end{array}$ \\
\hline $\mathrm{Rh}_{\mathrm{b}}^{(\mathrm{s})}$ & 2806 & 0.113 & $\begin{array}{c}-0.591 \\
-0.452 \\
0.446\end{array}$ & $\begin{array}{c}\nu_{\mathrm{OH}}^{b, s}+\nu_{\mathrm{OO}}^{s} \\
\nu_{\mathrm{OH}}^{b, a}+\nu_{\mathrm{OO}}^{A} \\
\nu_{\mathrm{OH}}^{b, s}\end{array}$ \\
\hline $\mathrm{Rh}_{\mathrm{c}}^{(\mathrm{s})}$ & 2626 & 0.138 & $\begin{array}{c}0.632 \\
0.547 \\
-0.547\end{array}$ & $\begin{array}{c}\nu_{\mathrm{OH}}^{b, s}+\nu_{\mathrm{OO}}^{s} \\
\nu_{\mathrm{OH}}^{b, s} \\
\nu_{\mathrm{OH}}^{b, a}+\nu_{\mathrm{OO}}^{A}\end{array}$ \\
\hline $\mathrm{Rh}_{\mathrm{d}}^{(\mathrm{s})}$ & 2178 & 0.301 & $\begin{array}{c}-0.628 \\
-0.608 \\
0.291\end{array}$ & $\begin{array}{c}\nu_{\mathrm{OH}}^{b, s} \\
\nu_{\mathrm{OH}}^{b, a}+\nu_{\mathrm{OO}}^{A} \\
\nu_{\mathrm{HOH}}^{b / f}+\nu_{\mathrm{rot}-\mathrm{C}}^{b / f}\end{array}$ \\
\hline $\mathrm{Rh}_{\mathrm{e}}^{(\mathrm{s})}$ & 2147 & 0.977 & $\begin{array}{l}-0.689 \\
-0.662 \\
-0.241\end{array}$ & $\begin{array}{c}\nu_{\mathrm{HOH}}^{b}+\nu_{\text {rot-C }}^{b / f} \\
\nu_{\mathrm{OH}}^{b, s}+\nu_{\mathrm{OO}}^{a} \\
\nu_{\text {rot-A }}^{b}+\nu_{\text {rot-B }}^{b}\end{array}$ \\
\hline $\mathrm{Rh}_{\mathrm{f}}^{(\mathrm{s})}$ & 2097 & 0.098 & $\begin{array}{l}-0.951 \\
-0.203 \\
-0.153\end{array}$ & $\begin{array}{c}\nu_{\mathrm{HOH}}^{b / f}+\nu_{\mathrm{rot}-\mathrm{C}}^{b / f} \\
\nu_{\mathrm{OH}}^{b, s} \\
\nu_{\mathrm{OH}}^{b, a}+\nu_{\mathrm{OO}}^{A}\end{array}$ \\
\hline $\mathrm{Rh}_{\mathrm{g}}^{(\mathrm{s})}$ & 2026 & 1.000 & $\begin{array}{c}0.635 \\
-0.462 \\
0.389\end{array}$ & $\begin{array}{c}\nu_{\mathrm{HOH}}^{b}+\nu_{\mathrm{rot}-\mathrm{C}}^{b / f} \\
\nu_{\mathrm{OH}}^{b, a} \\
\nu_{\mathrm{rot}-\mathrm{A}}^{b}+\nu_{\mathrm{rot}-\mathrm{B}}^{b}\end{array}$ \\
\hline $\mathrm{Rh}_{\mathrm{h}}^{(\mathrm{s})}$ & 1938 & 0.306 & $\begin{array}{c}0.871 \\
0.258 \\
-0.255\end{array}$ & $\begin{array}{c}\nu_{\mathrm{HOH}}^{w, s}+\nu_{\mathrm{OO}}^{A} \\
\nu_{\mathrm{OH}}^{b, a} \\
\nu_{\mathrm{HOH}}^{b}+\nu_{\mathrm{rot}-\mathrm{C}}^{b / f}\end{array}$ \\
\hline $\mathrm{Rh}_{\mathrm{i}}^{(\mathrm{s})}$ & 1793 & 0.828 & $\begin{array}{r}-0.775 \\
-0.448 \\
0.274\end{array}$ & $\begin{array}{c}\nu_{\mathrm{rot}-\mathrm{A}}^{b}+\nu_{\mathrm{rot}-\mathrm{B}}^{b} \\
\nu_{\mathrm{HOH}}^{b}+\nu_{\mathrm{OO}}^{A} \\
\nu_{\mathrm{HOH}}^{b / f}+\nu_{\mathrm{OO}}^{s}\end{array}$ \\
\hline $\mathrm{Rh}_{\mathrm{j}}^{(\mathrm{s})}$ & 1764 & 0.710 & $\begin{array}{c}0.844 \\
-0.386 \\
0.248\end{array}$ & $\begin{array}{c}\nu_{\mathrm{HOH}}^{b}+\nu_{\mathrm{OO}}^{A} \\
\nu_{\mathrm{rot}-\mathrm{A}}^{b}+\nu_{\mathrm{rot}-\mathrm{B}}^{b} \\
\nu_{\mathrm{OH}}^{b, a}\end{array}$ \\
\hline
\end{tabular}

${ }^{a}$ These features are plotted in red in Figure 3 and Figure S1. 
Table S11: Shifted, Coupled GSPA Frequencies, Relative Intensities, and Assignments for $\mathrm{D}_{7} \mathrm{O}_{3}{ }^{+a}(\mathrm{Rd})$

\begin{tabular}{|c|c|c|c|c|}
\hline Symbol & Frequency $\left(\mathrm{cm}^{-1}\right)$ & Rel. Int. & $C_{n}$ & $n$ \\
\hline \multirow[t]{3}{*}{$\mathrm{Rd}_{\mathrm{a}}^{(\mathrm{s})}$} & 2190 & 0.027 & -0.682 & $\nu_{\mathrm{OH}}^{b, s}+\nu_{\mathrm{OO}}^{A}$ \\
\hline & & & -0.571 & $\nu_{\mathrm{OH}}^{b, s}+\nu^{b / f}$ \\
\hline & & & 0.386 & $\nu_{\mathrm{OH}}^{b, A}$ \\
\hline \multirow[t]{3}{*}{$\mathrm{Rd}_{\mathrm{b}}^{(\mathrm{s})}$} & 2182 & 0.027 & -0.785 & $\nu_{\mathrm{OH}}^{b, s}+\nu_{\mathrm{rot}-\mathrm{C}}^{b / f}$ \\
\hline & & & 0.526 & $\nu_{\mathrm{OH}}^{b, s}+\nu_{\mathrm{OO}}^{A}$ \\
\hline & & & -0.252 & $\nu_{\mathrm{OH}}^{b, A}+\nu_{\mathrm{OO}}^{s}$ \\
\hline \multirow[t]{3}{*}{$\operatorname{Rd}_{\mathrm{c}}^{(\mathrm{s})}$} & 2177 & 0.049 & 0.583 & $\nu_{\mathrm{OH}}^{b, A}+\nu_{\mathrm{OO}}^{A}$ \\
\hline & & & -0.550 & $\nu_{\mathrm{OH}}^{b, s}+\nu_{\mathrm{OO}}^{s}$ \\
\hline & & & -0.468 & $\nu_{\mathrm{OH}}^{b, s}$ \\
\hline \multirow[t]{3}{*}{$\mathrm{Rd}_{\mathrm{d}}^{(\mathrm{s})}$} & 1692 & 0.202 & -0.736 & $\nu_{\mathrm{OH}}^{b, s}$ \\
\hline & & & 0.380 & $\nu_{\mathrm{HOH}}^{w, a}+\nu_{\mathrm{rot}-\mathrm{C}}^{b / f}$ \\
\hline & & & -0.354 & $\nu_{\mathrm{OH}}^{b, A}+\nu_{\mathrm{OO}}^{A}$ \\
\hline \multirow[t]{3}{*}{$\operatorname{Rd}_{\mathrm{e}}^{(\mathrm{s})}$} & 1650 & 1.000 & 0.816 & $\nu_{\mathrm{OH}}^{b, A}$ \\
\hline & & & 0.299 & $\nu_{\mathrm{OH}}^{b, s}+\nu_{\mathrm{OO}}^{A}$ \\
\hline & & & -0.267 & $\nu_{\mathrm{HOH}}^{w, a}+\nu_{\mathrm{OO}}^{s}$ \\
\hline \multirow[t]{3}{*}{$\operatorname{Rd}_{\mathrm{f}}^{(\mathrm{s})}$} & 1566 & 0.072 & 0.940 & $\nu_{\mathrm{HOH}}^{w, a}+\nu_{\mathrm{OO}}^{s}$ \\
\hline & & & 0.225 & $\nu_{\mathrm{OH}}^{b, A}$ \\
\hline & & & -0.122 & $\nu_{\mathrm{HOH}}^{b / f}+\nu_{\mathrm{OO}}^{s}$ \\
\hline \multirow[t]{3}{*}{$\mathrm{Rd}_{\mathrm{g}}^{(\mathrm{s})}$} & 1461 & 0.089 & -0.878 & $\nu_{\mathrm{HOH}}^{b}+\nu_{\mathrm{rot}-\mathrm{C}}^{b / f}$ \\
\hline & & & -0.403 & $\nu_{\mathrm{HOH}}^{b / f}+\nu_{\mathrm{OO}}^{s}$ \\
\hline & & & -0.146 & $\nu_{\mathrm{rot}-\mathrm{B}}^{b}+\nu_{\mathrm{rot}-\mathrm{B}}^{b}$ \\
\hline \multirow[t]{3}{*}{$\mathrm{Rd}_{\mathrm{h}}^{(\mathrm{s})}$} & 1393.8 & 0.472 & -0.823 & $\nu_{\mathrm{rot}-\mathrm{B}}^{b}+\nu_{\mathrm{rot}-\mathrm{B}}^{b}$ \\
\hline & & & -0.341 & $\nu_{\mathrm{HOH}}^{b / f}+\nu_{\mathrm{OO}}^{s}$ \\
\hline & & & -0.225 & $\nu_{\mathrm{OH}}^{b, a}$ \\
\hline
\end{tabular}

${ }^{a}$ These features are plotted in red in Figure 3 and Figure S1. 
Table S12: Coupled GSPA Frequencies, Relative Intensities, and Assignments for $\mathrm{H}_{9} \mathrm{O}_{4}^{+a}(\mathrm{Th})$

\begin{tabular}{|c|c|c|c|c|}
\hline Symbol & Frequency $\left(\mathrm{cm}^{-1}\right)$ & Rel. Int & $C_{n}$ & $n$ \\
\hline \multirow[t]{3}{*}{$\mathrm{Th}_{\mathrm{a}}^{(\mathrm{m})}$} & 3175 & 0.098 & -0.530 & $\nu_{\mathrm{OH}}^{b, a(2)}+\nu_{\mathrm{OO}}^{a(1)}$ \\
\hline & & & 0.529 & $\nu_{\mathrm{OH}}^{b, a(1)}+\nu_{\mathrm{OO}}^{a(2)}$ \\
\hline & & & 0.486 & $\nu_{\mathrm{OH}}^{b, a(1)}+\nu_{\mathrm{OO}}^{s}$ \\
\hline \multirow[t]{3}{*}{$\operatorname{Th}_{\mathrm{a}}^{(\mathrm{m})}$} & 3175 & 0.098 & 0.530 & $\nu_{\mathrm{OH}}^{b, a(2)}+\nu_{\mathrm{OO}}^{a(2)}$ \\
\hline & & & 0.530 & $\nu_{\mathrm{OH}}^{b, a(1)}+\nu_{\mathrm{OO}}^{a(1)}$ \\
\hline & & & -0.485 & $\nu_{\mathrm{OH}}^{b, a(2)}+\nu_{\mathrm{OO}}^{s}$ \\
\hline \multirow[t]{3}{*}{$\mathrm{Th}_{\mathrm{b}}^{(\mathrm{m})}$} & 2881 & 0.046 & 0.908 & $\nu_{\mathrm{OH}}^{b, s}+\nu_{\text {trans }, \mathrm{C}}^{b}$ \\
\hline & & & 0.395 & $\nu_{\mathrm{OH}}^{b, s}+\nu_{\mathrm{wag}}^{w, i}$ \\
\hline & & & -0.082 & $\nu_{\mathrm{OH}}^{b, a(2)}+\nu_{\mathrm{rock}}^{w, o(2)}$ \\
\hline \multirow[t]{3}{*}{$\mathrm{Th}_{\mathrm{c}}^{(\mathrm{m})}$} & 2713 & 0.081 & 0.676 & $\nu_{\mathrm{OH}}^{b, a(1)}+\nu_{\mathrm{OOO}}^{(2)}$ \\
\hline & & & -0.676 & $\nu_{\mathrm{OH}}^{b, a(2)}+\nu_{\mathrm{OOO}}^{(1)}$ \\
\hline & & & -0.238 & $\nu_{\mathrm{OH}}^{b, a(2)}$ \\
\hline \multirow[t]{3}{*}{$\mathrm{Th}_{\mathrm{d}}^{(\mathrm{m})}$} & 2722 & 0.075 & 0.685 & $\nu_{\mathrm{OH}}^{b, a(1)}+\nu_{\mathrm{OOO}}^{(1)}$ \\
\hline & & & 0.675 & $\nu_{\mathrm{OH}}^{b, a(2)}+\nu_{\mathrm{OOO}}^{(2)}$ \\
\hline & & & -0.229 & $\nu_{\mathrm{OH}}^{b, a(1)}$ \\
\hline \multirow[t]{3}{*}{$\mathrm{Th}_{\mathrm{e}}^{(\mathrm{m})}$} & 2558 & 1.000 & 0.686 & $\nu_{\mathrm{OH}}^{b, a(1)}$ \\
\hline & & & -0.531 & $\nu_{\mathrm{OH}}^{b, a(2)}$ \\
\hline & & & 0.211 & $\nu_{\mathrm{OH}}^{b, a(2)}+\nu_{\mathrm{OO}}^{a(2)}$ \\
\hline \multirow[t]{3}{*}{$\mathrm{Th}_{\mathrm{e}}^{(\mathrm{m})}$} & 2558 & 0.997 & 0.684 & $\nu_{\mathrm{OH}}^{b, a(2)}$ \\
\hline & & & 0.531 & $\nu_{\mathrm{OH}}^{b, a(1)}$ \\
\hline & & & -0.211 & $\nu_{\mathrm{OH}}^{b, a(2)}+\nu_{\mathrm{OO}}^{a(1)}$ \\
\hline
\end{tabular}

${ }^{a}$ These features are plotted in gold in Figure S1. 
Table S13: Coupled GSPA Frequencies, Relative Intensities, and Assignments for $\mathrm{D}_{9} \mathrm{O}_{4}^{+a}(\mathrm{Td})$

\begin{tabular}{|c|c|c|c|c|}
\hline Symbol & Frequency $\left(\mathrm{cm}^{-1}\right)$ & Rel. Int. & $C_{n}$ & $n$ \\
\hline \multirow[t]{3}{*}{$\mathrm{Td}_{\mathrm{a}}^{(\mathrm{m})}$} & 2399 & 0.022 & -0.651 & $\nu_{\mathrm{OH}}^{b, s}+\nu_{\mathrm{OO}}^{a(2)}$ \\
\hline & & & -0.429 & $\nu_{\mathrm{HOH}}^{w, a(1)}+\nu_{\mathrm{HOH}}^{w, s}$ \\
\hline & & & 0.367 & $\nu_{\mathrm{HOH}}^{w, a(2)}+\nu_{\mathrm{HOH}}^{w, a(2)}$ \\
\hline \multirow[t]{3}{*}{$\operatorname{Td}_{\mathrm{a}}^{(\mathrm{m})}$} & 2400 & 0.016 & -0.609 & $\nu_{\mathrm{HOH}}^{w, a(1)}+\nu_{\mathrm{HOH}}^{w, a(2)}$ \\
\hline & & & 0.563 & $\nu_{\mathrm{OH}}^{b, s}+\nu_{\mathrm{OO}}^{a(1)}$ \\
\hline & & & -0.422 & $\nu_{\mathrm{HOH}}^{w, a(2)}+\nu_{\mathrm{HOH}}^{w, s}$ \\
\hline \multirow[t]{3}{*}{$\mathrm{Td}_{\mathrm{b}}^{(\mathrm{m})}$} & 2389 & 0.024 & -0.706 & $\nu_{\mathrm{OH}}^{b, s}+\nu_{\mathrm{OO}}^{a(1)}$ \\
\hline & & & -0.584 & $\nu_{\mathrm{HOH}}^{w, a(2)}+\nu_{\mathrm{HOH}}^{w, s}$ \\
\hline & & & -0.318 & $\nu_{\mathrm{HOH}}^{w, a(1)}+\nu_{\mathrm{HOH}}^{w, a(2)}$ \\
\hline \multirow[t]{3}{*}{$\mathrm{Td}_{\mathrm{b}}^{(\mathrm{m})}$} & 2388 & 0.016 & 0.683 & $\nu_{\mathrm{HOH}}^{w, a(1)}+\nu_{\mathrm{HOH}}^{w, s}$ \\
\hline & & & -0.635 & $\nu_{\mathrm{OH}}^{b, s}+\nu_{\mathrm{OO}}^{a(2)}$ \\
\hline & & & -0.244 & $\nu_{\mathrm{HOH}}^{w, a(2)}+\nu_{\mathrm{HOH}}^{w, a(2)}$ \\
\hline \multirow[t]{3}{*}{$\operatorname{Td}_{\mathrm{c}}^{(\mathrm{m})}$} & 2378 & 0.015 & -0.705 & $\nu_{\mathrm{HOH}}^{w, a(1)}+\nu_{\mathrm{HOH}}^{w, a(2)}$ \\
\hline & & & 0.563 & $\nu_{\mathrm{HOH}}^{w, a(2)}+\nu_{\mathrm{HOH}}^{w, s}$ \\
\hline & & & -0.265 & $\nu_{\mathrm{OH}}^{b, a(2)}+\nu_{\mathrm{OO}}^{a(2)}$ \\
\hline \multirow[t]{3}{*}{$\mathrm{Td}_{\mathrm{c}}^{(\mathrm{m})}$} & 2376 & 0.014 & 0.570 & $\nu_{\mathrm{HOH}}^{w, a(2)}+\nu_{\mathrm{HOH}}^{w, a(2)}$ \\
\hline & & & -0.568 & $\nu_{\mathrm{HOH}}^{w, a(1)}+\nu_{\mathrm{HOH}}^{w, a(1)}$ \\
\hline & & & 0.456 & $\nu_{\mathrm{HOH}}^{w, a(1)}+\nu_{\mathrm{HOH}}^{w, s}$ \\
\hline \multirow[t]{3}{*}{$\mathrm{Td}_{\mathrm{d}}^{(\mathrm{m})}$} & 2119 & 0.034 & -0.558 & $\nu_{\mathrm{OH}}^{b, s}+\nu_{\mathrm{OOO}}^{(1)}$ \\
\hline & & & 0.499 & $\nu_{\mathrm{OH}}^{b, a(1)}+\nu_{\mathrm{OOO}}^{(1)}$ \\
\hline & & & -0.498 & $\nu_{\mathrm{OH}}^{b, a(2)}+\nu_{\mathrm{OOO}}^{(2)}$ \\
\hline \multirow[t]{3}{*}{$\mathrm{Td}_{\mathrm{d}}^{(\mathrm{m})}$} & 2118 & 0.031 & -0.634 & $\nu_{\mathrm{OH}}^{b, s}+\nu_{\mathrm{OOO}}^{(2)}$ \\
\hline & & & -0.441 & $\nu_{\mathrm{OH}}^{b, a(2)}+\nu_{\mathrm{OOO}}^{(1)}$ \\
\hline & & & 0.394 & $\nu_{\mathrm{HOH}}^{b(2)}+\nu_{\mathrm{HOH}}^{b(2)}$ \\
\hline \multirow[t]{3}{*}{$\mathrm{Td}_{\mathrm{e}}^{(\mathrm{m})}$} & 2019 & 0.046 & -0.981 & $\nu_{\mathrm{OH}}^{b, s}+\nu_{\text {trans }, \mathrm{C}}^{b}$ \\
\hline & & & 0.174 & $\nu_{\mathrm{OH}}^{b, s}+\nu_{\mathrm{wag}}^{w, i}$ \\
\hline & & & -0.066 & $\nu_{\mathrm{OH}}^{b, a(1)}+\nu_{\mathrm{wag}}^{w, o(2)}$ \\
\hline \multirow[t]{3}{*}{$\mathrm{Td}_{\mathrm{f}}^{(\mathrm{m})}$} & 2000 & 1.000 & -0.884 & $\nu_{\mathrm{OH}}^{b, a(2)}$ \\
\hline & & & -0.210 & $\nu_{\mathrm{OH}}^{b, s}+\nu_{\mathrm{OO}}^{a(1)}$ \\
\hline & & & -0.167 & $\nu_{\mathrm{HOH}}^{b(1)}+\nu_{\mathrm{HOH}}^{b(2)}$ \\
\hline \multirow{3}{*}{$\mathrm{Td}_{\mathrm{f}}^{(\mathrm{m})}$} & 1999 & 0.994 & 0.881 & $\nu_{\mathrm{OH}}^{b, a(1)}$ \\
\hline & & & -0.210 & $\nu_{\mathrm{OH}}^{b, s}+\nu_{\mathrm{OO}}^{a(2)}$ \\
\hline & & & -0.156 & $\nu_{\mathrm{OH}}^{b, a(2)}+\nu_{\mathrm{OO}}^{a(1)}$ \\
\hline
\end{tabular}

${ }^{a}$ These features are plotted in gold in Figure S1. 
Table S14: Coupled GSPA Frequencies, Relative Intensities, and Assignments for $\mathrm{H}_{7} \mathrm{O}_{3}{ }^{+a}(\mathrm{Rh})$

\begin{tabular}{|c|c|c|c|c|}
\hline Symbol & Frequency $\left(\mathrm{cm}^{-1}\right)$ & Rel. Int. & $C_{n}$ & $n$ \\
\hline $\mathrm{Rh}_{\mathrm{a}}^{(\mathrm{m})}$ & 2899 & 0.095 & $\begin{array}{c}0.913 \\
-0.416 \\
0.295\end{array}$ & $\begin{array}{c}\nu_{\mathrm{OH}}^{b, s}+\nu_{\mathrm{OO}}^{A} \\
\nu_{\mathrm{OH}}^{b, a} \\
\nu_{\mathrm{OH}}^{b, a}+\nu_{\mathrm{OO}}^{s}\end{array}$ \\
\hline $\mathrm{Rh}_{\mathrm{b}}^{(\mathrm{m})}$ & 2805 & 0.094 & $\begin{array}{l}-0.584 \\
-0.443 \\
-0.440\end{array}$ & $\begin{array}{c}\nu_{\mathrm{OH}}^{b, s}+\nu_{\mathrm{OO}}^{s} \\
\nu_{\mathrm{HOH}}^{b}+\nu_{\mathrm{HOH}}^{b} \\
\nu_{\mathrm{OH}}^{b, a}+\nu_{\mathrm{OO}}^{A}\end{array}$ \\
\hline $\mathrm{Rh}_{\mathrm{c}}^{(\mathrm{m})}$ & 2623 & 0.121 & $\begin{array}{c}-0.626 \\
0.558 \\
-0.546\end{array}$ & $\begin{array}{c}\nu_{\mathrm{OH}}^{b, s}+\nu_{\mathrm{OO}}^{s} \\
\nu_{\mathrm{OH}}^{b, a}+\nu_{\mathrm{OO}}^{A} \\
\nu_{\mathrm{OH}}^{b, s}\end{array}$ \\
\hline $\mathrm{Rh}_{\mathrm{d}}^{(\mathrm{m})}$ & 2168.1 & 0.279 & $\begin{array}{c}-0.641 \\
-0.595 \\
0.332\end{array}$ & $\begin{array}{c}\nu_{\mathrm{OH}}^{b, s} \\
\nu_{\mathrm{OH}}^{b, a}+\nu_{\mathrm{OO}}^{A} \\
\nu_{\mathrm{HOH}}^{b / f}+\nu_{\mathrm{rot}-\mathrm{C}}^{b / f}\end{array}$ \\
\hline $\mathrm{Rh}_{\mathrm{e}}^{(\mathrm{m})}$ & 2139 & 0.753 & $\begin{array}{l}0.772 \\
0.610 \\
0.216\end{array}$ & $\begin{array}{c}\nu_{\mathrm{HOH}}^{b}+\nu_{\text {rot-C }}^{b / f} \\
\nu_{\mathrm{OH}}^{b, a} \\
\nu_{\mathrm{OH}}^{b, s}+\nu_{\mathrm{OO}}^{A}\end{array}$ \\
\hline $\mathrm{Rh}_{\mathrm{f}}^{(\mathrm{m})}$ & 2096 & 0.104 & $\begin{array}{l}-0.937 \\
-0.236 \\
-0.176\end{array}$ & $\begin{array}{c}\nu_{\mathrm{HOH}}^{b / f}+\nu_{\mathrm{rot}-\mathrm{C}}^{b / f} \\
\nu_{\mathrm{OH}}^{b, s} \\
\nu_{\mathrm{OH}}^{b, a}+\nu_{\mathrm{OO}}^{A}\end{array}$ \\
\hline $\mathrm{Rh}_{\mathrm{g}}^{(\mathrm{m})}$ & 2005 & 0.859 & $\begin{array}{c}-0.712 \\
0.432 \\
-0.362\end{array}$ & $\begin{array}{c}\nu_{\mathrm{HOH}}^{w, a}+\nu_{\mathrm{OO}}^{s} \\
\nu_{\mathrm{OH}}^{b, a} \\
\nu_{\mathrm{HOH}}^{b}+\nu_{\mathrm{rot}-\mathrm{C}}^{b / f}\end{array}$ \\
\hline $\mathrm{Rh}_{\mathrm{h}}^{(\mathrm{m})}$ & 1988 & 0.335 & $\begin{array}{c}0.633 \\
-0.627 \\
-0.343\end{array}$ & $\begin{array}{c}\nu_{\mathrm{HOH}}^{w, a}+\nu_{\mathrm{OO}}^{s} \\
\nu_{\mathrm{HOH}}^{w, s}+\nu_{\mathrm{OO}}^{A} \\
\nu_{\mathrm{HOH}}^{b}+\nu_{\mathrm{rot}-\mathrm{C}}^{b / f}\end{array}$ \\
\hline $\mathrm{Rh}_{\mathrm{i}}^{(\mathrm{m})}$ & 1926 & 1.000 & $\begin{array}{c}-0.718 \\
-0.439 \\
0.376\end{array}$ & $\begin{array}{c}\nu_{\mathrm{HOH}}^{w, s}+\nu_{\mathrm{OO}}^{A} \\
\nu_{\mathrm{OH}}^{b, a} \\
\nu_{\mathrm{HOH}}^{b}+\nu_{\text {rotf }}^{b / f}\end{array}$ \\
\hline $\mathrm{Rh}_{\mathrm{j}}^{(\mathrm{m})}$ & 1769 & 0.138 & $\begin{array}{c}0.959 \\
-0.179 \\
0.145\end{array}$ & $\begin{array}{c}\nu_{\mathrm{HOH}}^{b}+\nu_{\mathrm{OO}}^{A} \\
\nu_{\mathrm{HOH}}^{b / f}+\nu_{\mathrm{OO}}^{s} \\
\nu_{\mathrm{OH}}^{b, a}\end{array}$ \\
\hline
\end{tabular}

${ }^{a}$ These features are plotted in gold in Figure S1. 
Table S15: Coupled GSPA Frequencies, Relative Intensities, and Assignments for $\mathrm{D}_{7} \mathrm{O}_{3}{ }^{+a}(\mathrm{Rd})$

\begin{tabular}{|c|c|c|c|c|}
\hline Symbol & Frequency $\left(\mathrm{cm}^{-1}\right)$ & Rel. Int. & $C_{n}$ & $n$ \\
\hline \multirow[t]{3}{*}{$\mathrm{Rd}_{\mathrm{a}}^{(\mathrm{m})}$} & 2190 & 0.025 & -0.674 & $\nu_{\mathrm{OH}}^{b, s}+\nu_{\mathrm{OO}}^{A}$ \\
\hline & & & -0.584 & $\nu_{\mathrm{OH}}^{b, s}+\nu_{\text {rot-C }}^{b / f}$ \\
\hline & & & 0.382 & $\nu_{\mathrm{OH}}^{b, t}$ \\
\hline \multirow{3}{*}{$\mathrm{Rd}_{\mathrm{b}}^{(\mathrm{m})}$} & 2182 & 0.027 & -0.776 & $\nu_{\mathrm{OH}}^{b, s}+\nu_{\text {rot-C }}^{b / f}$ \\
\hline & & & 0.537 & $\nu_{\mathrm{OH}}^{b, s}+\nu_{\mathrm{OO}}^{A}$ \\
\hline & & & -0.257 & $\mathrm{H}+\nu_{\mathrm{OO}}^{s}$ \\
\hline \multirow[t]{3}{*}{$\mathrm{Rd}_{\mathrm{c}}^{(\mathrm{m})}$} & 2177 & 0.048 & -0.584 & $\nu_{\mathrm{OH}}^{b, A}+\nu_{\mathrm{OO}}^{A}$ \\
\hline & & & 0.551 & $\nu_{\mathrm{OH}}^{b, s}+\nu_{\mathrm{OO}}^{s}$ \\
\hline & & & 0.467 & $\nu_{\mathrm{OH}}^{b, s}$ \\
\hline \multirow[t]{3}{*}{$\mathrm{Rd}_{\mathrm{d}}^{(\mathrm{m})}$} & 1691 & 0.195 & -0.732 & $\nu_{\mathrm{OH}}^{b, s}$ \\
\hline & & & 0.383 & $\nu_{\mathrm{HOH}}^{w, a}+\nu_{\mathrm{rot}-\mathrm{C}}^{b / f}$ \\
\hline & & & -0.351 & $\nu_{\mathrm{OH}}^{b, A}+\nu_{\mathrm{OO}}^{A}$ \\
\hline \multirow[t]{3}{*}{$\mathrm{Rd}_{\mathrm{e}}^{(\mathrm{m})}$} & 1645 & 1.000 & -0.804 & $\nu_{\mathrm{OH}}^{b, A}$ \\
\hline & & & 0.291 & $\nu_{\mathrm{HOH}}^{w, s}+\nu_{\mathrm{rot}-\mathrm{C}}^{b / f}$ \\
\hline & & & -0.289 & $\nu_{\mathrm{OH}}^{b, s}+\nu_{\mathrm{OO}}^{A}$ \\
\hline \multirow[t]{3}{*}{$\mathrm{Rd}_{\mathrm{f}}^{(\mathrm{m})}$} & 1565 & 0.098 & 0.932 & $\nu_{\mathrm{HOH}}^{w, a}+\nu_{\mathrm{OO}}^{s}$ \\
\hline & & & 0.251 & \\
\hline & & & -0.129 & $\nu_{\mathrm{HOH}}^{b / f}+\nu_{\mathrm{OO}}^{s}$ \\
\hline \multirow[t]{3}{*}{$\mathrm{Rd}_{\mathrm{g}}^{(\mathrm{m})}$} & 1460 & 0.121 & -0.839 & $10+\nu_{\mathrm{rot}-\mathrm{C}}^{b / f}$ \\
\hline & & & -0.477 & $\nu_{\mathrm{HOH}}^{b / f}+\nu_{\mathrm{OO}}^{s}$ \\
\hline & & & 0.145 & $\nu_{\mathrm{HOH}}^{w, s}+\nu_{\mathrm{OO}}^{A}$ \\
\hline \multirow[t]{3}{*}{$\mathrm{Rd}_{\mathrm{h}}^{(\mathrm{m})}$} & 1430 & 0.111 & -0.862 & $\nu_{\mathrm{HOH}}^{b / f}+\nu_{\mathrm{OO}}^{s}$ \\
\hline & & & 0.423 & $10+\nu_{\text {rot-C }}^{b / f}$ \\
\hline & & & -0.152 & $\nu_{\mathrm{O}}^{b,}$ \\
\hline \multirow[t]{2}{*}{$\mathrm{Rd}_{\mathrm{i}}^{(\mathrm{m})}$} & 1230 & 0.190 & 0.898 & $\nu_{\mathrm{rot}-\mathrm{B}}^{b}+\nu_{\mathrm{rot}-\mathrm{B}}^{b}$ \\
\hline & & & $\begin{array}{l}-0.328 \\
-0.204\end{array}$ & $\begin{array}{l}\nu_{\mathrm{HOH}}^{\omega, a}+\nu_{\mathrm{OOO}} \\
\nu_{\mathrm{HOH}}^{b / f}+\nu_{\mathrm{OOO}}\end{array}$ \\
\hline
\end{tabular}

${ }^{a}$ These features are plotted in gold in Figure S1. 


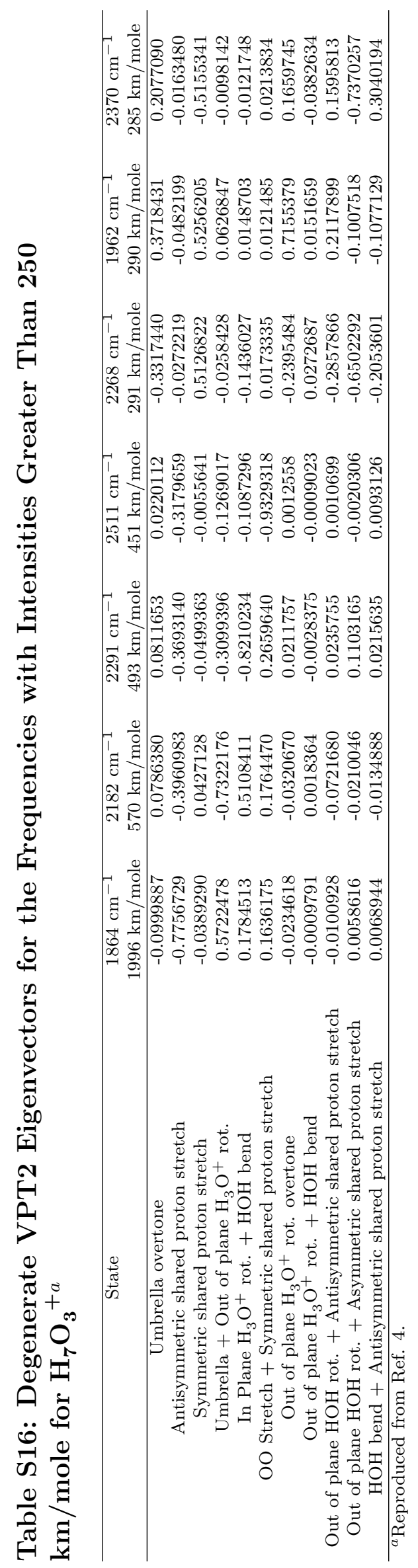




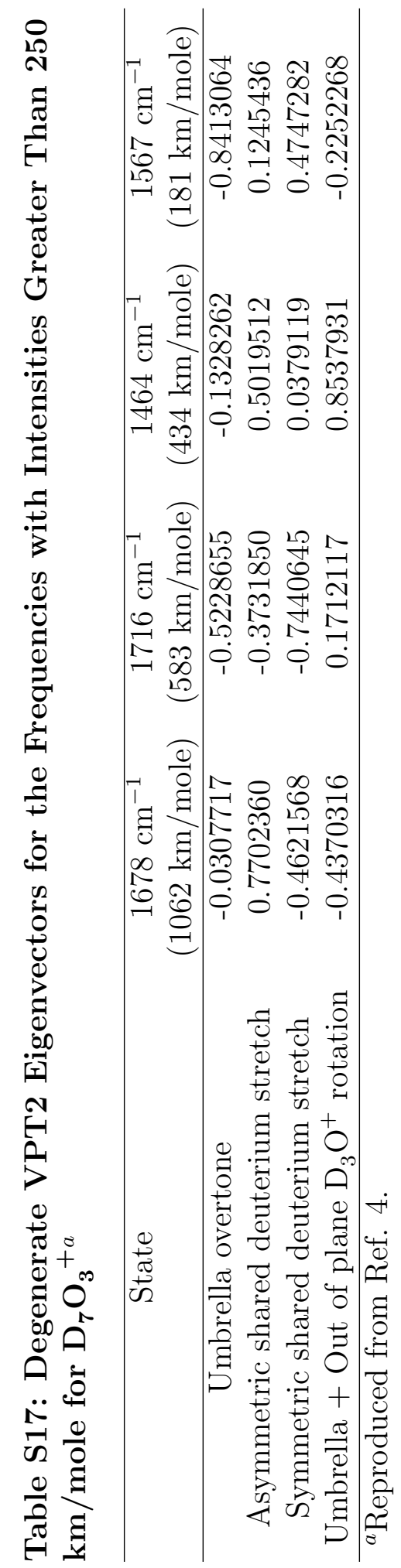




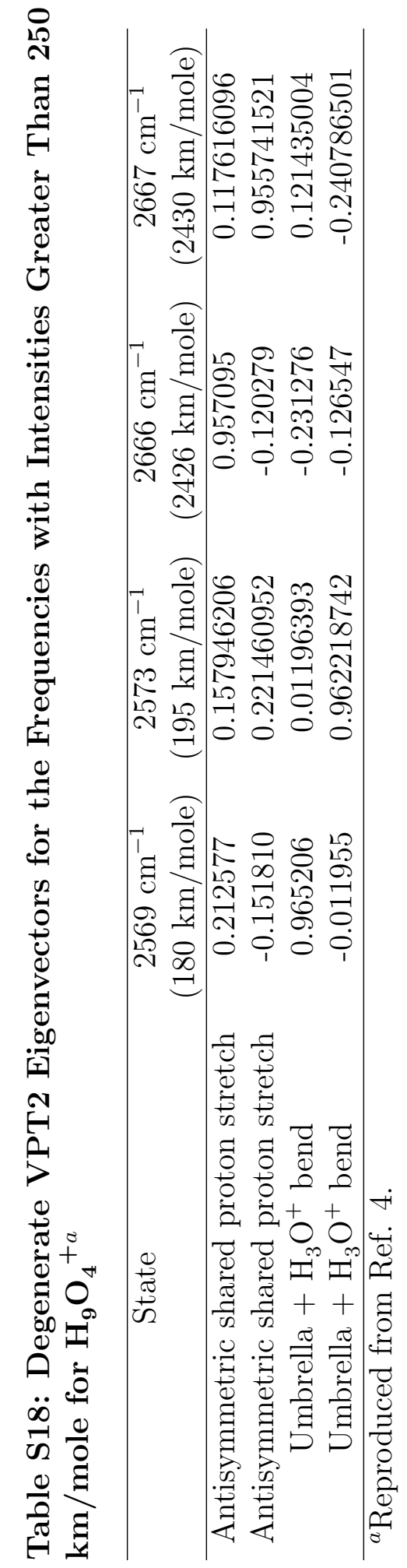


Table S19: GSPA Calculated Frequencies, Relative Intensities, and Assignments for the Low Frequency Region of $\mathrm{H}_{7} \mathrm{O}_{3}{ }^{+}(\mathrm{Rh})$ and $\mathrm{H}_{9} \mathrm{O}_{4}{ }^{+}(\mathrm{Th})^{a}$

\begin{tabular}{ccccc}
\hline System & Symbol & Frequency $\left(\mathrm{cm}^{-1}\right)$ & Rel. Int. & Assignment \\
\hline $\mathrm{H}_{7} \mathrm{O}_{3}{ }^{+}$ & $\mathrm{Rh}_{\mathrm{a}}^{(\mathrm{f})}$ & 820 & 0.304 & $\nu_{\text {rot-B }}^{b}$ \\
& $\mathrm{Rh}_{\mathrm{b}}^{(\mathrm{f})}$ & 450 & 0.344 & $\nu_{\text {wag }}^{w, o}+\nu_{\text {wag }}^{w, o}$ \\
& $\mathrm{Rh}_{\mathrm{c}}^{(\mathrm{f})}$ & 369 & 1.000 & $\nu_{\mathrm{OO}}^{a}$ \\
& $\mathrm{Rh}_{\mathrm{d}}^{(\mathrm{f})}$ & 325 & 0.878 & $\nu_{\text {rock }}^{w, i}$ \\
& $\mathrm{Rh}_{\mathrm{e}}^{(\mathrm{f})}$ & 253 & 0.227 & $\nu_{\text {rot-B }}^{f}+\nu_{\text {rot-B }}^{f}$ \\
$\mathrm{H}_{9} \mathrm{O}_{4}{ }^{+}$ & $\mathrm{Th}_{\mathrm{a}}^{(\mathrm{f})}$ & 827 & 0.419 & $\nu_{\text {umb }}^{b}$ \\
& $\mathrm{Th}_{\mathrm{b}}^{(\mathrm{f})}$ & 460 & 0.066 & $\nu_{\text {wag }}^{w, o(1)}+\nu_{\text {wag }}^{w, i}$ \\
& & 460 & 0.066 & $\nu_{\text {wag }}^{w, o(2)}+\nu_{\text {wag }}^{w, i}$ \\
& $\mathrm{Th}_{\mathrm{c}}^{(\mathrm{f})}$ & 358 & 0.467 & $\nu_{\mathrm{OO}}^{a(1)}$ \\
& & 358 & 0.467 & $\nu_{\mathrm{OO}}^{a(2)}$ \\
& $\mathrm{Th}_{\mathrm{d}}^{(\mathrm{f})}$ & 315 & 1.000 & $\nu_{\text {rock }}^{w, i}$ \\
& $\mathrm{Th}_{\mathrm{e}}^{(\mathrm{f})}$ & 245 & 0.172 & $\nu_{\mathrm{OO}}^{s}+\nu_{\text {trans,C }}^{b}$ \\
\hline
\end{tabular}

${ }^{a}$ These features are plotted in blue in Figure 4 . 


\section{References}

(1) Finney, J. M.; DiRisio, R. J.; McCoy, A. B. Guided Diffusion Monte Carlo: A Method for Studying Molecules and Ions That Display Large Amplitude Vibrational Motions. J. Phys. Chem. A 2020, 124, 9567-9577.

(2) Duong, C. H.; Gorlova, O.; Yang, N.; Kelleher, P. J.; Johnson, M. A.; McCoy, A. B.; Yu, Q.; Bowman, J. M. Disentangling the Complex Vibrational Spectrum of the Protonated Water Trimer, $\mathrm{H}^{+}\left(\mathrm{H}_{2} \mathrm{O}\right)_{3}$, with Two-Color IR-IR Photodissociation of the Bare Ion and Anharmonic VSCF/VCI Theory. J. Phys. Chem. Lett. 2017, 8, 3782-3789.

(3) Esser, T. K.; Knorke, H.; Asmis, K. R.; Schöllkopf, W.; Yu, Q.; Qu, C.; Bowman, J. M.; Kaledin, M. Deconstructing Prominent Bands in the Terahertz Spectra of $\mathrm{H}_{7} \mathrm{O}_{3}^{+}$and $\mathrm{H}_{9} \mathrm{O}_{4}^{+}$: Intermolecular Modes in Eigen Clusters. J. Phys. Chem. Lett. 2018, 9, 798-803.

(4) Dzugan, L. C. Theoretical Treatments of the Effects of Low Frequency Vibrations on $\mathrm{OH}$ Stretches in Molecules and Ion-Water Complexes That Undergo Large Amplitude Motions. Ph.D. thesis, The Ohio State University, 2017. 